\title{
Computational analysis of complement inhibitor compstatin using molecular dynamics
}

\author{
Didier Devaurs $^{1}$ (D) . Dinler A. Antunes ${ }^{2} \cdot$ Lydia E. Kavraki $^{2}$
}

Received: 21 January 2020 / Accepted: 14 July 2020 / Published online: 12 August 2020

(C) Springer-Verlag GmbH Germany, part of Springer Nature 2020

\begin{abstract}
The complement system plays a major role in human immunity, but its abnormal activation can have severe pathological impacts. By mimicking a natural mechanism of complement regulation, the small peptide compstatin has proven to be a very promising complement inhibitor. Over the years, several compstatin analogs have been created, with improved inhibitory potency. A recent analog is being developed as a candidate drug against several pathological conditions, including COVID19. However, the reasons behind its higher potency and increased binding affinity to complement proteins are not fully clear. This computational study highlights the mechanistic properties of several compstatin analogs, thus complementing previous experimental studies. We perform molecular dynamics simulations involving six analogs alone in solution and two complexes with compstatin bound to complement component 3 . These simulations reveal that all the analogs we consider, except the original compstatin, naturally adopt a pre-bound conformation in solution. Interestingly, this set of analogs adopting a pre-bound conformation includes analogs that were not known to benefit from this behavior. We also show that the most recent compstatin analog (among those we consider) forms a stronger hydrogen bond network with its complement receptor than an earlier analog.
\end{abstract}

Keywords Compstatin $\cdot$ Complement system $\cdot$ Complement inhibition $\cdot$ Molecular dynamics

\section{Introduction}

The complement system acts as the first line of defense in the immune system, as part of both innate and adaptive immunity [1]. It plays a major role in homeostasis by clearing foreign pathogens and compromised host cells [2]. The complement system is activated through several initiation pathways that produce strong opsonins, pro-inflammatory anaphylatoxins, and membrane attack complexes [3]. Complement activity is normally tightly regulated by various plasma and membrane proteins, such as complement receptor 1 and factor $\mathrm{H}$ [1]. Erroneous complement activation leads to tissue damage, causing or aggravating numerous

Lydia E. Kavraki

kavraki@rice.edu

1 University of Grenoble Alpes, CNRS, Inria, Grenoble INP, LJK, 38000, Grenoble, France

2 Department of Computer Science, Rice University, 6100 Main St, Houston, TX 77005, USA pathological conditions, such as heart attacks, strokes, burn injuries, Alzheimer's disease, adult respiratory distress syndrome, and various autoimmune diseases [2, 4]. To prevent or remedy these undesirable effects, several complement inhibitors have been developed, targeting different steps of complement activation $[3,5,6]$.

Thanks to its excellent efficacy, high specificity, low molecular weight, and ability to inhibit complement regardless of the initiation pathway, compstatin is considered a very promising complement inhibitor [3]. It has been evaluated for its potential to modulate the complement system in degenerative diseases. For example, it has been the subject of in vitro studies, non-human primate (NHP) studies, and clinical trials for age-related macular degeneration (AMD), but unfortunately without much success [7-10]. Compstatin has prevented lung fibrosis and organ damage in NHP models of $E$. coli sepsis [11, 12]. It has also proven effective against paroxysmal nocturnal hemoglobinuria $(\mathrm{PNH})$ and C3 glomerulopathy $(\mathrm{C} 3 \mathrm{G})$ in in vitro models $[13,14]$. In NHP models, it has reduced inflammation and bone loss during treatment of periodontitis, both in a ligature-induced disease setting and in a natural setting [15, 16]. Other NHP studies have shown that a single dose of compstatin prior 
to a hemodialysis session could alleviate hemodialysisinduced inflammation throughout the treatment [17]. Therefore, it could also prove useful against conditions related to innate immunity activation triggered by biomaterials during medical procedures [18]. Recently, compstatin has been successfully used to treat a patient suffering from acute respiratory distress syndrome (ARDS) triggered by COVID19 [19]. As a result, it is now being evaluated in a phase II clinical study [20].

Various compstatin analogs were developed over the years, with increased complement inhibition at every step [9, 21-26]. The mechanisms underlying the improved potency of each analog involve various factors that have been partially uncovered in several experimental studies (see Background section). In addition, structural models of some of these analogs, either free in solution or bound to a complement protein, have been deposited in the Protein Data Bank (PDB) [27]. However, it is not yet fully clear why recent compstatin analogs are more potent than their predecessors (see Background section).

In this paper, we perform a computational analysis of several compstatin analogs, using molecular dynamics (MD). We run MD simulations of six analogs free in solution and two complexes with compstatin bound to the core portion of C3-like complement proteins (see Background section). Our results are consistent with the view that one of the factors increasing binding affinity is the tendency of recent compstatin analogs to adopt a pre-bound conformation in solution [25]. However, we demonstrate that this tendency appeared in the lineage of compstatin analogs earlier than previously thought. In addition, we show that the latest compstatin analog (among the six we study) forms a stronger hydrogen bond network with complement proteins than an earlier analog. In this regard, our computational study complements previous experimental studies on explaining differences in behavior between compstatin analogs. This is important because compstatin is currently being developed as a drug candidate against various pathological conditions $[2,3]$.

\section{Background}

\section{Complement system}

The main ways to activate the complement system are the classical, lectin, and alternative pathways [1]. These recognition and activation pathways all converge at an amplification step involving the complement component 3 (C3) [2]. C3 is a large protein that belongs to the $\alpha_{2}$ macroglobulin family of host-defense molecules [1]. It is composed of 1641 amino acid residues, for a total molecular mass of $187 \mathrm{kDa}$. Upon activation by foreign pathogens or altered host cells via any of the pathways, enzymatic C3 convertase complexes are formed. Then, they transform the abundant plasma protein $\mathrm{C} 3$, through proteolytic cleavage, into its reactive fragments $\mathrm{C} 3 \mathrm{a}(9 \mathrm{kDa})$ and $\mathrm{C} 3 \mathrm{~b}(177$ $\mathrm{kDa}$ ) [28]. The opsonin $\mathrm{C} 3 \mathrm{~b}$ then attaches covalently to activating surfaces, where it can form additional C3 convertases, thereby amplifying the complement response and inducing downstream events such as pro-inflammatory signaling and phagocytosis [3].

The structure of native $\mathrm{C} 3$ was solved by X-ray crystallography at $3.3 \AA$ resolution [28]. It reveals a $\beta$ chain (residues 1-645) and an $\alpha$-chain (residues 650-1641) of 75 and $110 \mathrm{kDa}$, respectively, arranged in 13 domains. Most of the $\beta$-chain, i.e., residues $1-534$, form five socalled macroglobulin (MG) domains, denoted by MG1 to MG5. The sixth domain of the $\beta$-chain (residues 578-645) is called the linker (LNK) and runs in between domains MG1, MG4, and MG5, through the ring formed by domains MG1-MG5. The $\alpha$-chain also forms six domains by itself, but one of the additional MG domains, denoted by MG6, is formed by parts of both the $\alpha$-chain (residues 746-806) and the $\beta$-chain (residues 535-577). The core of $\mathrm{C} 3$, which is formed by domains MG1 to MG6 and LNK, is referred to as the $\beta$-ring and is known to be structurally stable [28]. That is why we will use it in our MD simulations (see Methods section).

\section{Complement inhibitor compstatin}

Compstatin is a small, cyclic, non-immunogenic peptide inhibiting the activation and amplification of the complement system [4]. It does so by binding to C3, sterically hindering the binding between substrate $\mathrm{C} 3$ and convertase complexes, and blocking C3's proteolytic activation [2]. Note that this happens without major alteration to the conformation of C3 [29]. In addition, compstatin binds to several proteins derived from $\mathrm{C} 3$, such as $\mathrm{C} 3 \mathrm{~b}$ and $\mathrm{C} 3 \mathrm{c}$ [4]. Interestingly, it was shown that its binding is improved by a naturally occurring point mutation of C3-like proteins observed in patients with a complement dysfunction [30].

Compstatin had been initially identified as a 13residue disulfide-bridged peptide with a molecular mass of $1551 \mathrm{Da}$ [4]. Its original amino acid sequence was Ile1-[Cys2-Val3-Val4-Gln5-Asp6-Trp7-Gly8-His9-His10-

Arg11-Cys12]-Thr13- $\mathrm{NH}_{2}$, where the brackets represent the disulfide bridge between Cys2 and Cys12. Its structure in solution was then determined using 2D nuclear magnetic resonance (NMR) techniques and deposited in the PDB under code 1A1P [31]. This structure was described as a closed-loop coil with a flexible type I $\beta$-turn comprising residues Gln5-Gly8. In what follows, we refer to this original compstatin as 1A1P. 
The acetylation of the amino-terminus was followed by a threefold increase in activity for the analog Accompstatin [21]. Then, the substitution of Val by the aromatic residue Trp at position 4 and of His by the helix promoter Ala at position 9 produced an ana$\log$ with a 45-fold higher inhibitory activity [21]. We refer to this analog as $4 \mathbf{W 9 A}$ because of the replacements Val4Trp and His9Ala (i.e., V4W and H9A). Its full sequence is Ac-Ile1-[Cys2-Val3-Trp4-Gln5-Asp6-Trp7Gly8-Ala9-His10-Arg11-Cys12]-Thr13-NH2. MD simulations of $4 \mathrm{~W} 9 \mathrm{~A}$ in solution showed that it had a reduced tendency to feature the Gln5-Gly8 $\beta$-turn characterizing 1A1P [32]. It was also hypothesized that its increased flexibility could explain its higher affinity. The crystal structure of 4W9A in complex with C3c (available under PDB code 2QKI) later showed that the compstatin-binding site is formed by domains MG4 and MG5, at the bottom end of the $\beta$-ring, far from other binding sites of C3 [29]. Comparing this bound conformation with the one in solution (i.e., 1A1P) reveals that compstatin undergoes a large conformational change upon binding [29]. The $\beta$ turn involves residues Gly8-Arg11 in bound compstatin, as opposed to residues Gln5-Gly8 in free compstatin. Overall, free compstatin seems to adopt an elongated and open $v$-shaped conformation, while bound compstatin seems to adopt a twisted and more closed $\alpha$-shaped conformation (see Fig. 1). After superposition, the root mean square deviation (RMSD) between these two conformations is $3.7 \AA$, considering only $\mathrm{C} \alpha$ atoms [29].

Several tryptophan analogs were then evaluated at position 4. Replacing Trp4 by a 1-methyl-tryptophan, i.e., $\operatorname{Trp}(1 \mathrm{Me})$ or $\operatorname{Trp}(\mathrm{Me})$ for short, produced a compstatin analog with a 264-fold increase in inhibitory activity over the original compstatin [22]. Its full sequence is Ac-Ile1-[Cys2-Val3-Trp(Me)4-Gln5-Asp6-Trp7-Gly8-Ala9His10-Arg11-Cys12]-Thr13-NH2. In what follows, we refer to this analog as $\mathbf{4 M e W}$, although it has also been designated as POT-4 (by Potentia Pharmaceuticals) and AL-78898A (by Alcon) in past clinical trials for exudative AMD [25]. Its increase in potency and binding affinity has been attributed to more favorable hydrophobic interactions mediated by the methylated tryptophan [22].

A follow-up substitution study yielded new analogs with improved efficacy and affinity. Replacing Gly by Sar (i.e., Gly with an N-methylation, or Gly(NMe) for short) at position 8 and of Thr by Ile at position 13 produced the analog known as Cp10, with increased kinetic association rate and binding entropy [23]. It is described as Ac-Ile1-[Cys2-Val3-Trp(Me)4-Gln5-Asp6-Trp7-Sar8-Ala9His10-Arg11-Cys12]-Ile13-NH2. It was shown that the N-methylation of Gly8 improved hydrophobic interactions and stabilized the bound-like $\beta$-turn structure [23]. This suggested that the improved binding affinity of Cp10 might be due to a higher similarity between its free and bound conformations, leading to faster ligand recognition and complex formation. This was later supported by the solution structure of Cp10 elucidated using NMR (which has not been deposited in the PDB) [25]. It is more compact than the solution structure of the original compstatin because of the presence of two $\beta$ turns involving residues Cys2-Gln5 and $\operatorname{Trp}(\mathrm{Me}) 4-\operatorname{Trp} 7$ (see Fig. 1).

The N-methylation of Ile (denoted by Ile(Me) for short) at position 13 yielded the analog known as Cp20, with an improved dissociation rate [23]. Its sequence is Ac-Ile1-[Cys2-Val3-Trp(Me)4-Gln5-Asp6-Trp7-Sar8Ala9-His10-Arg11-Cys12]-Ile(Me)13-NH2. Cp20 shows a 1000-fold increase in inhibitory potency and binding affinity for C3 compared to the original compstatin [23]. However, the high flexibility of residue 13 makes it difficult to elucidate the mechanisms underlying this improved activity and affinity.
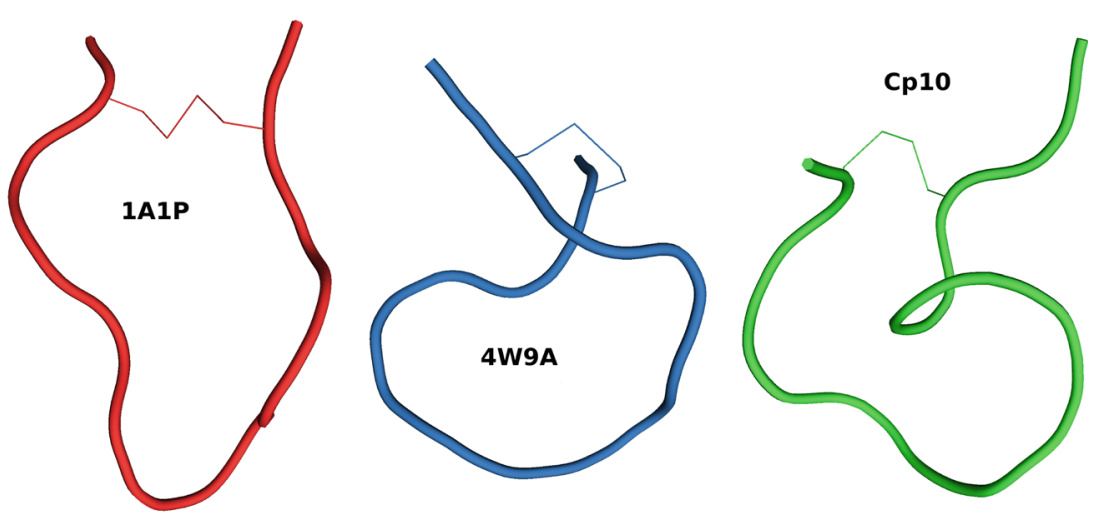

Fig. 1 Backbone conformations of compstatin analogs 1A1P, 4W9A, and $\mathrm{Cp} 10$. The conformation of 1A1P (free in solution) is elongated and $v$-shaped. The conformation of 4W9A (bound to $\mathrm{C} 3 \mathrm{c}$, under PDB code $2 \mathrm{QKI}$ ) is more compact and $\alpha$-shaped. The conformation of Cp10 (free in solution, extracted from an NMR ensemble) is more compact than that of $1 \mathrm{~A} 1 \mathrm{P}$. The disulfide bridge creating the cycle is represented with thin lines in all analogs 
Table 1 Amino acid sequences of the compstatin analogs that have been analyzed in this study

\begin{tabular}{|c|c|c|c|c|c|c|c|c|c|c|c|c|c|c|c|}
\hline & 0 & 1 & 2 & 3 & 4 & 5 & 6 & 7 & 8 & 9 & 10 & 11 & 12 & 13 & \\
\hline $1 \mathrm{~A} 1 \mathrm{P}$ & & I & $\mathrm{C}$ & V & $\mathrm{V}$ & Q & D & W & G & $\mathrm{H}$ & $\mathrm{H}$ & $\mathrm{R}$ & $\mathrm{C}$ & $\mathrm{T}$ & $\mathrm{NH}_{2}$ \\
\hline 4W9A & Ac & I & $\mathrm{C}$ & V & $\mathbf{W}$ & Q & D & W & G & $\mathbf{A}$ & $\mathrm{H}$ & $\mathrm{R}$ & $\mathrm{C}$ & $\mathrm{T}$ & $\mathrm{NH}_{2}$ \\
\hline $4 \mathrm{MeW}$ & Ac & I & $\mathrm{C}$ & V & W(Me) & Q & D & W & G & A & $\mathrm{H}$ & $\mathrm{R}$ & $\mathrm{C}$ & $\mathrm{T}$ & $\mathrm{NH}_{2}$ \\
\hline Cp10 & Ac & I & $\mathrm{C}$ & V & $\mathrm{W}(\mathrm{Me})$ & Q & D & W & Sar & A & $\mathrm{H}$ & $\mathrm{R}$ & $\mathrm{C}$ & I & $\mathrm{NH}_{2}$ \\
\hline $\mathrm{Cp} 20$ & $\mathrm{Ac}$ & I & $\mathrm{C}$ & V & $\mathrm{W}(\mathrm{Me})$ & Q & D & W & Sar & A & $\mathrm{H}$ & $\mathrm{R}$ & $\mathrm{C}$ & $\mathbf{I}(\mathbf{M e})$ & $\mathrm{NH}_{2}$ \\
\hline Сp40 & D-Y & I & $\mathrm{C}$ & V & $\mathrm{W}(\mathrm{Me})$ & $\mathrm{Q}$ & D & W & Sar & A & $\mathrm{H}$ & $\mathrm{R}$ & $\mathrm{C}$ & $\mathrm{I}(\mathrm{Me})$ & $\mathrm{NH}_{2}$ \\
\hline
\end{tabular}

Residues that were modified between two analogs are highlighted in bold font

The effects of replacing the N-terminal acetyl moiety (called position 0 for consistency of residue numbering with other analogs) by amino acids, including nonproteinogenic ones, were subsequently investigated [25]. The analog involving the D-isoform of tyrosine (DTyr) showed the highest inhibitory potency and the slowest dissociation rate. Its full amino acid residue sequence is DTyr0-Ile1-[Cys2-Val3-Trp(Me)4-Gln5-Asp6-Trp7-Sar8-

Ala9-His10-Arg11-Cys12]-Ile(Me)13-NH2. This analog also featured an improved solubility and more favorable pharmacokinetic properties. It was the first analog with sub-nanomolar binding affinity $\left(K_{D}=0.5 \mathrm{nM}\right.$, which is 5600-fold stronger than the original compstatin) and is known as Cp40. It is currently developed as a candidate drug by Amyndas (under the designation AMY-101) and has received orphan drug status from the European Medicines Agency and the US Food and Drug Administration for both $\mathrm{PNH}$ and $\mathrm{C} 3 \mathrm{G}[33,34]$. This analog has recently been used to successfully cure a patient suffering from ARDS resulting from COVID-19 pneumonia [19]. It is currently the subject of a phase II clinical study [20].

\section{Methods}

\section{Structural models of compstatin analogs}

We ran MD simulations of the six compstatin analogs (free in solution) presented in the Background section, whose amino acid sequences are recapitulated in Table 1. This required obtaining or creating all-atom structural models of these analogs. For the original compstatin analog, we used the first of the 21 NMR models of free compstatin reported under PDB code 1A1P. For 4W9A, we extracted chain $\mathrm{G}$ from the crystal structure of compstatin bound to $\mathrm{C} 3 \mathrm{c}$ (reported under PDB code $2 \mathrm{QKI}$ ). For $4 \mathrm{MeW}$, we constructed in silico a mutant by performing methylation of the tryptophan at position 4 in 4W9A using UCSF Chimera [35]. For Cp10, we obtained the first model from the undeposited NMR ensemble ${ }^{1}$ of this analog alone in solution [25]. For $\mathrm{Cp} 20$ and $\mathrm{Cp} 40$, we obtained the unpublished crystal structures ${ }^{1}$ of these analogs bound to $\mathrm{C} 3 \mathrm{~b}$, and extracted the relevant chains. For all these analogs, hydrogen atoms were added to the structural model using UCSF Chimera [35].

\section{Structural models of complexes involving compstatin}

Instead of modeling the entire protein $\mathrm{C} 3$, we chose to model only its $\beta$-ring (see Background section). This is a reasonable choice in the context of this study, for several reasons. First, compstatin is known to bind domains MG4 and MG5 of the $\beta$-ring without yielding any significant structural change in C3 [29]. Second, the structure of the $\beta$-ring is known to be very stable, as we observed when simulating it alone in solution. Third, the $\beta$-ring is conserved in all C3-like proteins to which compstatin binds. Observations collected from simulating compstatin binding to the $\beta$-ring alone can thus be generalized to all these proteins.

Even when restricting ourselves to using only the $\beta$ ring (and not the whole $\mathrm{C} 3$ protein), MD simulations are still very computationally expensive. Therefore, instead of simulating complexes involving all the compstatin analogs presented in the Background section, we chose to simulate only two complexes involving compstatin analogs 4W9A and $\mathrm{Cp} 40$. The rationale for this choice is that we mostly wanted to try and pinpoint mechanistic aspects of the higher binding affinity of the most potent analog, $\mathrm{Cp} 40$, in comparison to an older analog. This choice was also driven by the availability of crystal structures of compstatin bound to C3-like proteins. More specifically, to create the model involving 4W9A and the $\beta$-ring, we used the crystal structure of compstatin bound to $\mathrm{C} 3 \mathrm{c}$ (under PDB code 2QKI) [29]. To create the complex featuring Cp40, we used

\footnotetext{
${ }^{1} 2015$ communication from D Ricklin and JD Lambris
} 
an unpublished crystal structure ${ }^{1}$ of compstatin bound to C3b. In both cases, we extracted chain A and residues 746804 in chain $\mathrm{B}$, to form the $\beta$-ring, as well as chain $\mathrm{G}$, which is compstatin.

\section{Molecular dynamics simulations}

All simulations were run with the GROMACS v4.6.5 package [36] using the parameter set of the CHARMM27 force field [37], as well as the SPC water model. A cubic box was defined with $1.5 \mathrm{~nm}$ of liquid layer around the modeled structure, with periodic boundary conditions. Sodium $(\mathrm{Na}+)$ and chloride $(\mathrm{Cl}-)$ counter-ions were added to neutralize each system, with a final concentration of $0.15 \mathrm{~mol} / \mathrm{L}$. Temperature and pressure coupling were controlled by the algorithms v-rescale (with tau- $t=0.1 \mathrm{ps}$ ) and parrinello-rahman (with tau-p $=2 \mathrm{ps}$ ), respectively. A cutoff value of $1.2 \mathrm{~nm}$ was used for both the van der Waals and Coulomb interactions, using fast particlemesh Ewald electrostatics. Input MD files compatible with CHARMM27 were created with SwissParam [38] for compstatin analogs, and with pdb2gmx (from the GROMACS package) for the protein receptor (i.e., C3's $\beta$ ring). For the two complexes, input files were created by merging the files of compstatin and the $\beta$-ring.

Simulations were conducted following a previously described protocol [39]. Briefly, the production stage of the simulation was preceded by three steps of energy minimization (EM) and eight steps of equilibration (EQ). The first EM step was conducted using the steepestdescent algorithm and position restraints on the heavy atoms of the substrate $\left(5000 \mathrm{~kJ}^{-1} \mathrm{~mol}^{-1} \mathrm{~nm}^{-1}\right)$, allowing for relaxation of the solvent only. The second step used the same algorithm, without restraint; the third one used the conjugate-gradient algorithm without restraint. The EQ phase started at a temperature of $310 \mathrm{~K}$, for $300 \mathrm{ps,}$ with position restraints on the substrate's heavy atoms (5000 $\mathrm{kJ}^{-1} \mathrm{~mol}^{-1} \mathrm{~nm}^{-1}$ ), allowing for the formation of solvation layers. Then, temperature was reduced to $280 \mathrm{~K}$, and position restraints were gradually reduced. Then, temperature was progressively increased again, up to $300 \mathrm{~K}$. These EQ steps constituted the first 500 ps of each MD
Fig. 2 Root mean square deviation (RMSD) to compstatin's closed $\alpha$-shaped conformation (defined by the crystal structure of 4W9A bound to $\mathrm{C} 3 \mathrm{c}$, as reported under PDB code $2 \mathrm{QKI}$ ), for each compstatin analog. The RMSD (considering only $\mathrm{C} \alpha$ atoms) is reported in $\AA$ for 667 frames extracted from a $200 \mathrm{~ns}$ MD simulation of each analog alone in solution
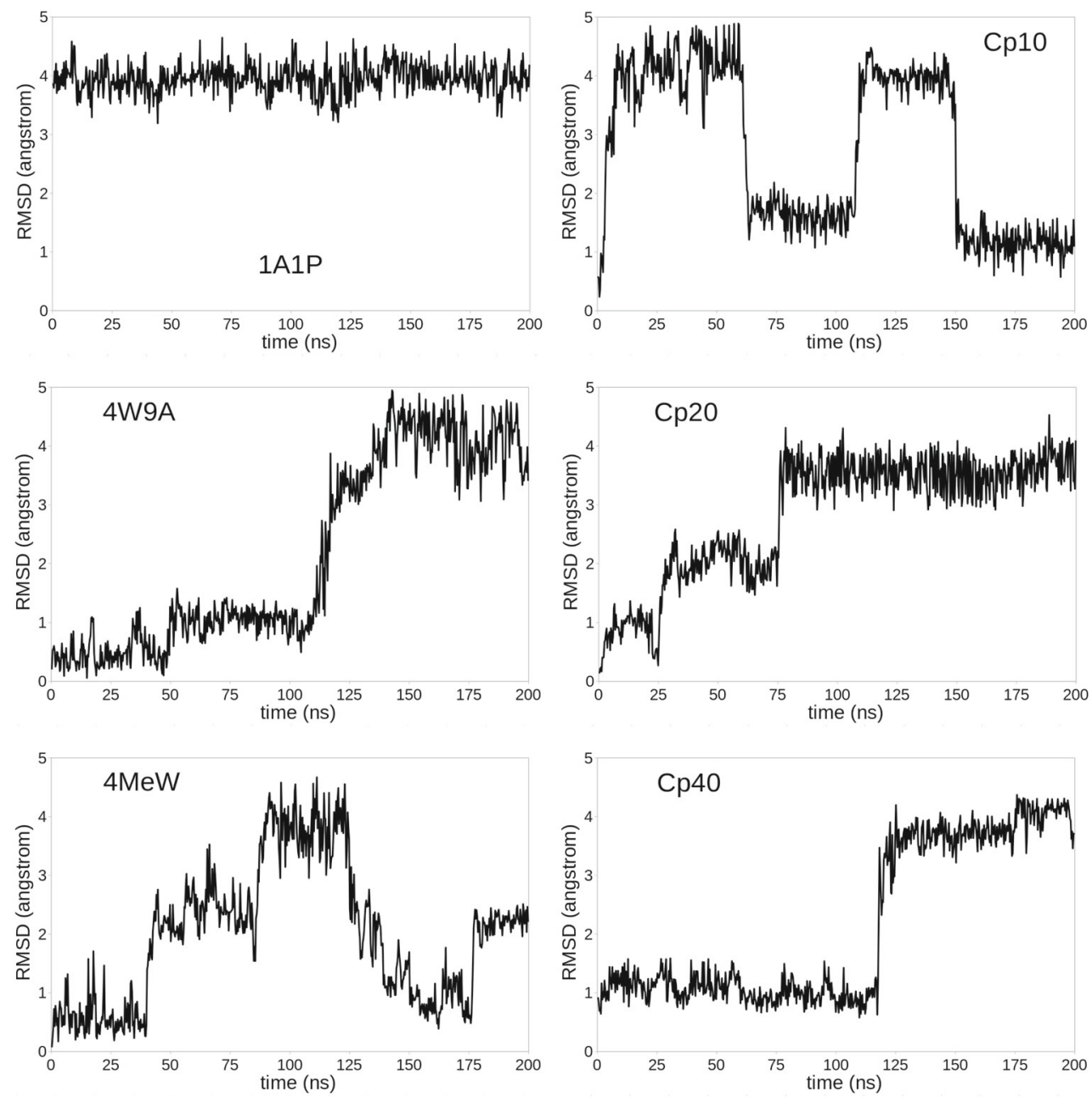
Fig. 3 Schematic representation of the time spent in each state (open $v$-shaped state, closed $\alpha$-shaped state, or intermediate state) during the $200 \mathrm{~ns}$ MD simulation of every compstatin analog, free in solution

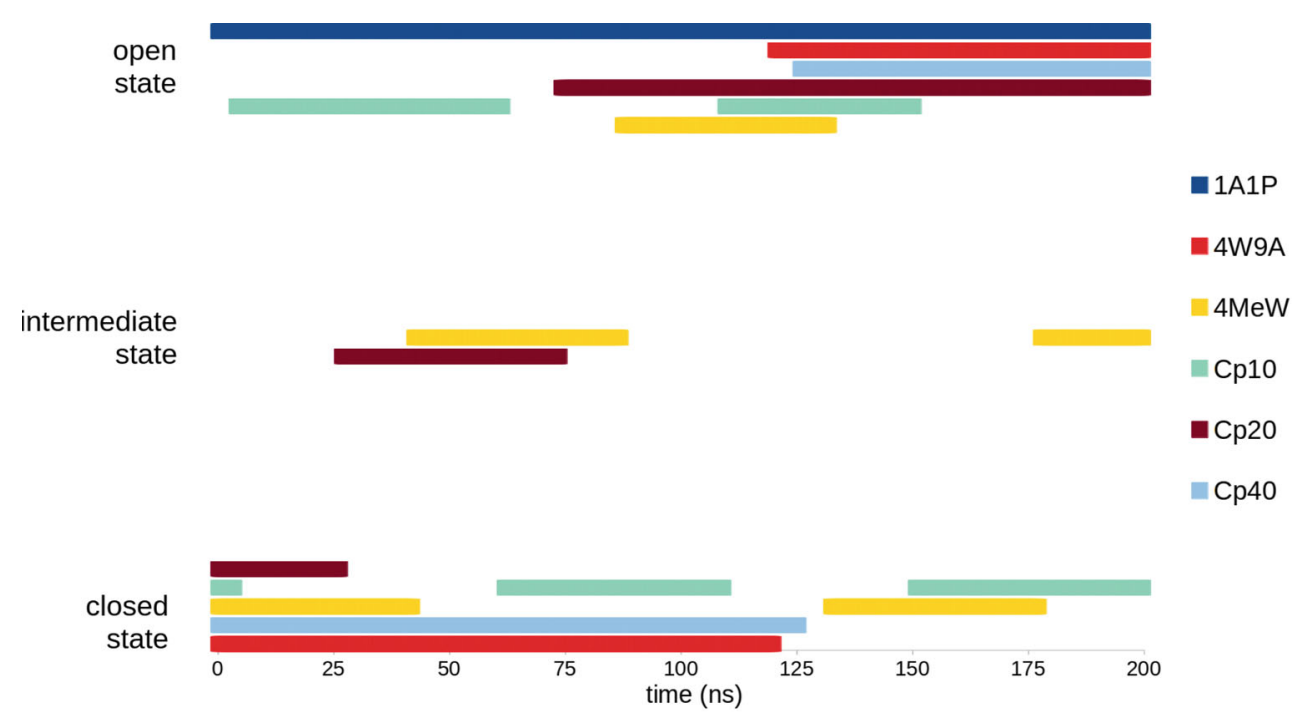

simulation. The production stage ran for $200 \mathrm{~ns}$ (for free compstatin) or $100 \mathrm{~ns}$ (for the two complexes) at constant temperature $(300 \mathrm{~K})$, without restraint.

After the simulation and post-processing steps, visual inspection of the trajectories was performed with VMD [40] and xmgrace. $^{2}$ Substrate conformations were extracted at regular steps using trjconv (from the GROMACS v4.6.5 package) [36]. The occupancy of each hydrogen bond during the MD simulation was calculated with the plot_hbmap.pl script. ${ }^{3}$

\section{Results}

\section{Free compstatin}

We ran a $200 \mathrm{~ns}$ MD simulation for the six compstatin analogs, free in solution, starting from a minimized version of their all-atom models (see Methods section). Note that, for analogs 1A1P and $\mathrm{Cp} 10$, the simulation starts from a "real" free conformation, while for the other analogs, the simulation starts from a bound-like conformation because their structural models were obtained by extracting compstatin from co-crystallized complexes. From each MD trajectory, we extract 667 frames, using a 0.3 ns timestep. We align each conformation of compstatin with the closed $\alpha$-shaped conformation of 4W9A (as reported under PDB code $2 \mathrm{QKI}$ ) and calculate the $\mathrm{C} \alpha$ RMSD between these two conformations.

The evolution of this RMSD to the closed $\alpha$-shaped state (measured in $\AA$ ) along the MD trajectory is reported

\footnotetext{
${ }^{2}$ http://plasma-gate.weizmann.ac.il/Grace

${ }^{3}$ http://www.bevanlab.biochem.vt.edu/Pages/Personal/justin/scripts. html
}

for each compstatin analog in Fig. 2. These plots show that, except for 1A1P, which always remains in its open $v$-shaped conformation, free compstatin usually switches (sometimes even back and forth) between its open $v$-shaped conformation and its closed $\alpha$-shaped conformation, and that it can adopt intermediate conformations, as illustrated by $4 \mathrm{MeW}$ and $\mathrm{Cp} 20$. This information is schematically represented in Fig. 3, for easier comparison between analogs. It shows that, after excluding 1A1P, Cp20 is the analog that spent the least amount of simulation time in its closed $\alpha$-shaped state, while all other analogs spent a similar amount of simulation time in their closed $\alpha$-shaped states, albeit not necessarily in a continuous fashion.

\section{Bound compstatin}

We ran a $100 \mathrm{~ns}$ MD simulation for compstatin analogs 4W9A and Cp40 in complex with the $\beta$-ring of C3like proteins, starting from minimized versions of all-atom models derived from co-crystal structures (see Methods section). From both MD trajectories, we extract 1000 frames, between 2 and $100 \mathrm{~ns}$, using a 0.098-ns timestep. Then, we perform the following analyses: First, we remove compstatin from each frame, align the remaining $\beta$-ring with that in the first frame, and calculate the C $\alpha$ RMSD between them. Plotting this RMSD over simulation time corroborates that the $\beta$-ring's conformation did not change in either MD simulation (data not shown). Second, we align compstatin in each frame with that in the first frame and calculate the $\mathrm{C} \alpha$ RMSD between them. Plots of this RMSD over simulation time confirm that both compstatin analogs remained in their closed $\alpha$-shaped state during the MD simulation (data not shown).

To assess differences in the binding of 4W9A and Cp40 to the $\beta$-ring, we monitor the inter-molecular hydrogen 
bonds that form during each MD simulation. To quantify the strength and importance of each hydrogen bond, we calculate its occupancy, i.e., the percentage of frames in which it is observed. Results are reported in Table 2 for hydrogen bonds whose occupancy is greater than 5\%.

Overall, hydrogen bond networks formed by $\mathrm{Cp} 40$ and 4W9A are quite similar. Two of the strongest bonds, with occupancy close to $100 \%$, are observed for both analogs: those between the tryptophan residues in compstatin, at positions 4 and 7, and residues Gly345 and Met457 in the $\beta$-ring, respectively. Two other strong bonds are common to both analogs: the one between C3's Met457 and compstatin's Gln5, with occupancy above 90\%, and the one between C3's Arg456 and compstatin's Trp4, with occupancy above $80 \%$. Six weaker bonds with an occupancy around 50\% and among which a lot of "switching" is observed are also common: they form between compstatin's Ala9 / His10 and C3's Asp491.

We can also observe differences between the hydrogen bond networks associated with $\mathrm{Cp} 40$ and 4W9A. First, a bond with acceptor in C3's Asn390 and that switches between compstatin's Cys2 (occupancy: 77\%) and Trp4 (occupancy: 15\%) in 4W9A, is stabilized toward Cys2 in $\mathrm{Cp} 40$, with occupancy close to $100 \%$. Second, a very weak bond with a donor in C3's Asn390 and acceptor in compstatin's Cys2, with an occupancy of $10 \%$ in 4W9A, is replaced by a stronger bond involving D-Tyr0, with an occupancy of $61 \%$ in Cp40. On the other hand, two weak bonds have seen their occupancy reduced when comparing 4W9A to Cp40: the one between compstatin's Gln5 and C3's Leu455, whose occupancy has decreased from 46 to $31 \%$, and the one between C3's Arg459 and compstatin's Asp6, whose occupancy has decreased from 35 to $7 \%$.

\section{Discussion}

In previous experimental studies on compstatin, it was suggested that the improved binding affinity of the most recent analogs was partially due to their ability to adopt in solution a conformation similar to their bound state (i.e., a closed $\alpha$-shaped conformation), therefore leading to a faster complex formation with C3-like proteins [25]. Our MD simulations of free compstatin confirm this view, as all analogs except the original one, seem to spend a significant amount of time in a closed $\alpha$-shaped state (cf. Fig. 3). At some point, this behavior had been attributed to the $\mathrm{N}$ methylation of compstatin's backbone (at position 8), which differentiates analogs $\mathrm{Cp} 10, \mathrm{Cp} 20$, and $\mathrm{Cp} 40$ from previous ones [25]. Our MD simulations do not support this theory, as the behavior of $4 \mathrm{~W} 9 \mathrm{~A}$ and $4 \mathrm{MeW}$ does not differ from that of the later analogs (cf. Fig. 3). The tendency of free

Table 2 Inter-molecular hydrogen bonds observed during the MD simulations of compstatin analogs Cp40 and 4W9A bound to C3's $\beta$-ring

\begin{tabular}{|c|c|c|c|c|c|c|c|c|c|}
\hline \multicolumn{5}{|l|}{$\mathrm{Cp} 40$} & \multicolumn{5}{|l|}{ 4W9A } \\
\hline \multicolumn{2}{|l|}{ Donor } & \multicolumn{2}{|l|}{ Acceptor } & \multirow{2}{*}{$\begin{array}{l}\text { Occupancy } \\
(\%)\end{array}$} & \multicolumn{2}{|l|}{ Donor } & \multicolumn{2}{|c|}{ Acceptor } & \multirow{2}{*}{$\begin{array}{l}\text { Occupancy } \\
(\%)\end{array}$} \\
\hline Residue & Atom & Residue & Atom & & Residue & Atom & Residue & Atom & \\
\hline M-Trp4 & $\mathrm{N}$ & Gly345 & $\mathrm{O}$ & 99 & Trp4 & $\mathrm{N}$ & Gly345 & $\mathrm{O}$ & 99 \\
\hline Cys2 & $\mathrm{N}$ & Asn390 & OD1 & 97 & Trp7 & NE1 & Met457 & $\mathrm{O}$ & 99 \\
\hline Trp7 & NE1 & Met457 & $\mathrm{O}$ & 96 & Met457 & $\mathrm{N}$ & Gln5 & OE1 & 97 \\
\hline Met457 & $\mathrm{N}$ & Gln5 & OE1 & 90 & Arg456 & $\mathrm{NE}$ & Trp4 & $\mathrm{O}$ & 81 \\
\hline $\operatorname{Arg} 456$ & $\mathrm{NE}$ & M-Trp4 & $\mathrm{O}$ & 80 & Cys2 & $\mathrm{N}$ & Asn390 & OD1 & 77 \\
\hline Asn390 & ND2 & D-Tyr0 & $\mathrm{O}$ & 61 & Ala9 & $\mathrm{N}$ & Asp491 & OD1 & 54 \\
\hline His10 & ND1 & Asp491 & OD2 & 57 & His 10 & ND1 & Asp491 & OD2 & 52 \\
\hline His 10 & $\mathrm{~N}$ & Asp491 & OD2 & 53 & His 10 & $\mathrm{~N}$ & Asp491 & OD2 & 51 \\
\hline Ala9 & $\mathrm{N}$ & Asp491 & OD1 & 51 & His 10 & $\mathrm{~N}$ & Asp491 & OD1 & 51 \\
\hline His 10 & ND1 & Asp491 & OD1 & 49 & His 10 & ND1 & Asp491 & OD1 & 47 \\
\hline His 10 & $\mathrm{~N}$ & Asp491 & OD1 & 49 & Gln5 & NE2 & Leu455 & $\mathrm{O}$ & 46 \\
\hline Ala9 & $\mathrm{N}$ & Asp491 & OD2 & 44 & Ala9 & $\mathrm{N}$ & Asp491 & OD2 & 43 \\
\hline Gln5 & NE2 & Leu455 & $\mathrm{O}$ & 31 & Arg459 & NH1 & Asp6 & $\mathrm{O}$ & 35 \\
\hline \multirow[t]{2}{*}{$\operatorname{Arg} 459$} & NH1 & Asp6 & $\mathrm{O}$ & 7 & Trp4 & NE1 & Asn390 & OD1 & 15 \\
\hline & & & & & Asn390 & ND2 & Cys2 & $\mathrm{O}$ & 10 \\
\hline
\end{tabular}

For each hydrogen bond, we report its donor and acceptor atoms (and the residues they belong to) as well as its occupancy (i.e., the percentage of MD frames in which it is observed). Residues with numbers between 2 and 10 belong to compstatin; the others belong to the $\beta$-ring. Only hydrogen bonds with an occupancy above $5 \%$ are listed 
compstatin to adopt a closed $\alpha$-shaped conformation seems to have appeared with the creation of 4W9A, most likely as a result of the amino acid substitutions it underwent. This is consistent with a previous MD study that had concluded that the higher affinity of 4W9A might be linked to its increased flexibility [32], although no comparison could be done with the bound conformation, which was released only later.

Our MD simulations of free compstatin also suggest that the improved binding affinity of the most recent analogs is not due to an increase in the tendency of free compstatin to spend time in its closed $\alpha$-shaped state. Indeed, the amount of time spent in that state is absolutely not correlated with the binding affinity of an analog. This is best illustrated by $\mathrm{Cp} 20$, which spent little time in its closed $\alpha$-shaped conformation (cf. Fig. 3), despite being the second most potent analog in this study.

Our MD simulations of bound 4W9A and Cp40 highlight some of the mechanisms underlying the higher binding affinity of Cp40. Despite forming relatively similar hydrogen bond networks with C3's $\beta$-ring, 4W9A and Cp40 present several noteworthy differences. Most notably, Cp40 forms an additional strong hydrogen bond that replaces an equivalent yet weaker bond in 4W9A. Interestingly, the $\mathrm{D}$-Tyr0 residue that was added to $\mathrm{Cp} 40$ is involved in a relatively stable hydrogen bond that replaces an equivalent but very weak bond involving Cys 2 in 4W9A. The stronger hydrogen bond network formed by $\mathrm{Cp} 40$ when binding to C3-like proteins is certainly not sufficient to explain Cp40's stronger binding affinity on its own, but it certainly has a significant impact.

Despite their benefits, MD simulations are mostly limited to short time-scale interactions. However, interactions between C3-like proteins and compstatin might present sharper differences at longer time-scales. To study this possibility, we will carry out coarse-grained simulations of complexes involving compstatin, such as those we performed on C3-like proteins alone [41, 42]. One of the challenges will be to select force fields that can correctly assess the energetic contributions of this small peptide, especially for analogs comprising unusual amino acids.

Acknowledgments The authors are extremely thankful to John Lambris and Daniel Ricklin for sharing data on the structural models of several compstatin analogs. All molecular structures are depicted by images produced with the PyMOL Molecular Graphics System, Version 1.8 Schrödinger, LLC. PyMOL was also used to align molecular structures and calculate RMSD between them. Visual inspection and modeling of compstatin analogs also involved UCSF Chimera [35], developed by the Resource for Biocomputing, Visualization, and Informatics at the University of California, San Francisco (supported by NIGMS P41-GM103311).

Author contributions All authors contributed to the study conception and design. Computational modeling and simulations, as well as data collection and analysis were performed by Didier Devaurs and Dinler Antunes. The first draft of the manuscript was written by Didier
Devaurs and all authors commented on subsequent versions of the manuscript. All authors read and approved the final manuscript.

Funding information This work was supported in part by the National Science Foundation (NSF) under Grant CCF 1423304, and by Rice University Funds. Computational simulations were run on equipment supported in part by the Data Analysis and Visualization Cyberinfrastructure funded by NSF under Grant OCI 0959097, on equipment supported by the Cyberinfrastructure for Computational Research funded by NSF under Grant CNS 0821727, as well as on equipment supported in part by the Big-Data Private-Cloud Research Cyberinfrastructure MRI-award funded by NSF under grant CNS 1338099 and by Rice University.

Data availability Crystal structures used in this study are available in the Protein Data Bank (PDB) [27]. Other structural models and ensembles produced by molecular dynamics simulations are available from the corresponding author, L.K., upon request.

\section{Compliance with ethical standards}

Conflict of interests The authors declare that they have no conflict of interest.

Ethical approval This article does not contain any studies with human participants or animals performed by any of the authors.

\section{References}

1. Gros P, Milder FJ, Janssen BJ (2008) Complement driven by conformational changes. Nat Rev Immunol 8(1):48-58

2. Morgan BP, Harris CL (2015) Complement, a target for therapy in inflammatory and degenerative diseases. Nat Rev Drug Discov 14:857-877

3. Ricklin D, Lambris JD (2016) New milestones ahead in complement-targeted therapy. Semin Immunol 28(3):208-222

4. Sahu A, Kay BK, Lambris JD (1996) Inhibition of human complement by a C3-binding peptide isolated from a phagedisplayed random peptide library. J Immunol 157(2):884-891

5. Kocsis A, Kékesi KA, Szász R, Végh BM, Balczer J, Dobó J, et al. (2010) Selective inhibition of the lectin pathway of complement with phage display selected peptides against mannose-binding lectin-associated serine protease (MASP)-1 and -2: significant contribution of MASP-1 to lectin pathway activation. J Immunol 185(7):4169-4178

6. Mohan RR, Wilson M, Gorham RD, Harrison RE, Morikis VA, Kieslich CA, et al. (2018) Virtual screening of chemical compounds for discovery of complement C3 ligands. ACS Omega 3(6):6427-6438

7. Alcon licenses complement pathway inhibitor for macular degeneration (2009) Nat Rev Drug Discov 8(12):922

8. Chi ZL, Yoshida T, Lambris JD, Iwata T (2010) Suppression of drusen formation by compstatin, a peptide inhibitor of complement C3 activation, on cynomolgus monkey with earlyonset macular degeneration. In: Lambris J, Adamis A (eds) Inflammation and retinal disease: complement biology and pathology. vol. 703 of advances in experimental medicine and biology. Springer, New York, pp 127-135 
9. Gorham RD, Forest DL, Tamamis P, López de Victoria A, Kraszni M, Kieslich CA, et al. (2013) Novel compstatin family peptides inhibit complement activation by drusen-like deposits in human retinal pigmented epithelial cell cultures. Exp Eye Res 116:96-108

10. Leung E, Landa G (2013) Update on current and future novel therapies for dry age-related macular degeneration. Expert Rev Clin Pharmacol 6(5):565-579

11. Silasi-Mansat R, Zhu H, Popescu NI, Peer G, Sfyroera G, Magotti $\mathrm{P}$, et al. (2010) Complement inhibition decreases the procoagulant response and confers organ protection in a baboon model of Escherichia coli sepsis. Blood 116(6):1002-1010

12. Silasi-Mansat R, Zhu H, Georgescu C, Popescu N, Keshari RS, Peer G, et al. (2015) Complement inhibition decreases early fibrogenic events in the lung of septic baboons. J Cell Mol Med. 19(11):2549-2563

13. Risitano AM, Ricklin D, Huang Y, Reis ES, Chen H, Ricci P et al (2014) Peptide inhibitors of C3 activation as a novel strategy of complement inhibition for the treatment of paroxysmal nocturnal hemoglobinuria. Blood 123(13):2094-2101

14. Zhang Y, Shao D, Ricklin D, Hilkin BM, Nester CM, Lambris JD, et al. (2015) Compstatin analog Cp40 inhibits complement dysregulation in vitro in $\mathrm{C} 3$ glomerulopathy. Immunobiology 220(8):993-998

15. Maekawa T, Abe T, Hajishengallis E, Hosur KB, DeAngelis RA, Ricklin D, et al. (2014) Genetic and intervention studies implicating complement $\mathrm{C} 3$ as a major target for the treatment of periodontitis. J Immunol 192(12):6020-6027

16. Maekawa T, Briones RA, Resuello RR, Tuplano JV, Hajishengallis E, Kajikawa T, et al. (2016) Inhibition of pre-existing natural periodontitis in non-human primates by a locally administered peptide inhibitor of complement C3. J Clin Periodontol 43(3):238-249

17. Reis ES, DeAngelis RA, Chen H, Resuello RR, Ricklin D, Lambris JD (2015) Therapeutic C3 inhibitor Cp40 abrogates complement activation induced by modern hemodialysis filters. Immunobiology 220(4):476-482

18. Ekdahl KN, Lambris JD, Elwing H, Ricklin D, Nilsson PH, Teramura Y, et al. (2011) Innate immunity activation on biomaterial surfaces: a mechanistic model and coping strategies. Adv Drug Deliv Rev 63(12):1042-1050

19. Mastaglio S, Ruggeri A, Risitano AM, Angelillo P, Yancopoulou D, Mastellos DC, et al. (2020) The first case of COVID-19 treated with the complement C3 inhibitor AMY-101. Clin Immunol 215:108450

20. Amyndas initiates phase 2 trial of complement inhibitor AMY-101 in COVID-19 (2020). www.amyndas.com/covid-19. Accessed 7 Jul 2020

21. Mallik B, Katragadda M, Spruce LA, Carafides C, Tsokos CG, Morikis D, et al. (2005) Design and NMR characterization of active analogues of compstatin containing non-natural amino acids. J Med Chem 48(1):274-286

22. Katragadda M, Magotti P, Sfyroera G, Lambris JD (2006) Hydrophobic effect and hydrogen bonds account for the improved activity of a complement inhibitor, compstatin. J Med Chem 49(15):4616-4622

23. Qu H, Magotti P, Ricklin D, Wu EL, Kourtzelis I, Wu YQ, et al. (2011) Novel analogues of the therapeutic complement inhibitor compstatin with significantly improved affinity and potency. Mol Immunol 48(4):481-489

24. Tamamis P, López de Victoria A, Gorham RD, Bellows-Peterson ML, Pierou P, Floudas CA, et al. (2012) Molecular dynamics in drug design: new generations of compstatin analogs. Chem Biol Drug Des 79(5):703-718

25. Qu H, Ricklin D, Bai H, Chen H, Reis ES, Maciejewski M, et al. (2013) New analogs of the clinical complement inhibitor compstatin with subnanomolar affinity and enhanced pharmacokinetic properties. Immunobiology 218(4):496-505
26. Gorham RD, Forest DL, Khoury GA, Smadbeck J, Beecher CN, Healy ED, et al. (2015) New compstatin peptides containing Nterminal extensions and non-natural amino acids exhibit potent complement inhibition and improved solubility characteristics. J Med Chem 58(2):814-826

27. Berman HM, Westbrook J, Feng Z, Gilliland G, Bhat TN, Weissig $\mathrm{H}$, et al. (2000) The protein data bank. Nucleic Acids Res 28(1):235-242

28. Janssen BJ, Huizinga EG, Raaijmakers HC, Roos A, Daha MR, Nilsson-Ekdahl K, et al. (2005) Structures of complement component $\mathrm{C} 3$ provide insights into the function and evolution of immunity. Nature 437(7058):505-511

29. Janssen BJ, Halff EF, Lambris JD, Gros P (2007) Structure of compstatin in complex with complement component $\mathrm{C} 3 \mathrm{c}$ reveals a new mechanism of complement inhibition. J Biol Chem 282(40):29241-29247

30. Sfyroera G, Ricklin D, Reis ES, Chen H, Wu EL, Kaznessis YN, et al. (2015) Rare loss-of-function mutation in complement component C3 provides insight into molecular and pathophysiological determinants of complement activity. J Immunol 194(7):3305-3316

31. Morikis D, Assa-Munt N, Sahu A, Lambris JD (1998) Solution structure of Compstatin, a potent complement inhibitor. Protein Sci 7(3):619-627

32. Tamamis P, Skourtis SS, Morikis D, Lambris JD, Archontis G (2007) Conformational analysis of compstatin analogues with molecular dynamics simulations in explicit water. J Mol Graph 26(2):571-580

33. Reis ES, Mastellos DC, Yancopoulou D, Risitano AM, Ricklin D, Lambris JD (2015) Applying complement therapeutics to rare diseases. Clin Immunol 161(2):225-240

34. Press release: Amyndas' lead candidate AMY-101 receives orphan drug status from the FDA and the EMA for the treatment of $\mathrm{C} 3$ glomerulopathy (2016). http://c3g.today/?p=305. Accessed 5 Jul 2019)

35. Pettersen EF, Goddard TD, Huang CC, Couch GS, Greenblatt DM, Meng EC, et al. (2004) UCSF chimera-a visualization system for exploratory research and analysis. J Comput Chem 25(13):16051612

36. Pronk S, Páll S, Schulz R, Larsson P, Bjelkmar P, Apostolov R, et al. (2013) GROMACS 4.5: a high-throughput and highly parallel open source molecular simulation toolkit. Bioinformatics 29(7):845-854

37. Bjelkmar P, Larsson P, Cuendet MA, Hess B, Lindahl E (2010) Implementation of the CHARMM force field in GROMACS: analysis of protein stability effects from correction maps, virtual interaction sites, and water models. J Chem Theory Comput 6(2):459-466

38. Zoete V, Cuendet MA, Grosdidier A, Michielin O (2011) Swissparam: a fast force field generation tool for small organic molecules. J Comput Chem 32(11):2359-2368

39. Devaurs D, Papanastasiou M, Antunes DA, Abella JR, Moll M, Ricklin D, et al. (2018) Native state of complement protein C3d analysed via hydrogen exchange and conformational sampling. Int J Comput Biol Drug Des 11(1-2):90-113

40. Humphrey W, Dalke A, Schulten K (1996) VMD Visual molecular dynamics. J Mol Graph 14(1):33-38

41. Devaurs D, Antunes DA, Papanastasiou M, Moll M, Ricklin D, Lambris JD (2017) Coarse-grained conformational sampling of protein structure improves the fit to experimental hydrogenexchange data. Front Mol Biosci 4(13)

42. Devaurs D, Antunes DA, Kavraki LE (2018) Revealing unknown protein structures using computational conformational sampling guided by experimental hydrogen-exchange data. Int J Mol Sci 19(11):3406

Publisher's note Springer Nature remains neutral with regard to jurisdictional claims in published maps and institutional affiliations. 